УДК 669.86: 536.25

\title{
Thermovibrational Low-Mode Model of Convection in a Horizontal Layer with Longitudinal Vibrations
}

\author{
Vadim A. Sharifulin* \\ Perm National Research Politechnic University \\ Pozdeeva, 11/B, Perm, 614990
}

Russia

Received 02.10.2016, received in revised form 10.01.2017, accepted 20.02.2017

\begin{abstract}
Thermovibrational convection in a horizontal layer of fluid between isothermal solid boundaries heated to different temperatures in the presence of longitudinal vibrations is considered in this paper. Stability and supercritical bifurcation of convection is investigated in a low-mode approximation. Bifurcation diagrams of supercritical modes are analytically obtained in the area of stability of supercritical convection. The analysis of diagrams shows that vibrations can lead to the rigid type of the occurrence of convection when upper boundary is heated. In addition, the hysteresis between stationary states is observed. The size of hysteresis interval of the Rayleigh numbers increases with the growth of the Gershuni number. A numerical study of the linear stability of the supercritical vibration-convective flows in the interval of Prandtl numbers $1 \leqslant \operatorname{Pr} \leqslant 10$ is conducted in the context of the proposed model. The region of flow stability decreases with increasing the Prandtl number. For any value of the Prandtl number from the given interval drastic excitation of stationary vibrational convection with hysteresis is possible.
\end{abstract}

Keywords: thermovibrational convection, low-mode model, flat layer, hard excitation, hysteresis. DOI: 10.17516/1997-1397-2017-10-2-158-169.

The study of supercritical regimes of thermal convection in a plane horizontal layer between the solid isothermal planes heated to different temperatures and subjected to longitudinal vibration is of considerable interest. It would help to understand the nucleation mechanism in terms of the laws of the bifurcation of complex convective phenomena. It is also of importance in obtaining new materials. In the absence of vibration this problem is the classical problem of Rayleigh convection in a horizontal layer heated from below [1-3]. This is the most studied problem of thermal convection. Supercritical mode of convection is investigated in numerous works both theoretically and experimentally when the Rayleigh number $R a$ exceeds the critical value $R a_{c r}=1708$. Supercritical convection under the action of longitudinal vibrations in the case of zero-gravity has been studied in [4]. Nonlinear supercritical vibrational convection was investigated numerically by finite difference method when the Prandtl number is equal to one and the Gershuni number $G s$ exceeds the critical value $G s_{c r}=2129$ [5] (in papers [6,7] the Gershuni number was called the vibrational Rayleigh number).

When vibrational and gravitional mechanisms of the convection are present the linear stability of the state of averaged mechanical equilibrium was studied only theoretically. It follows from the linear theory of stability [5] that the set of Rayleigh and Gershuni numbers, for which the state of averaged mechanical equilibrium is neutrally stable, is defined by the following relation

$$
R a / R a_{c r}+G s / G s_{c r}=1 .
$$

*vadim.sharifulin@pstu.ru

(c) Siberian Federal University. All rights reserved 
When the left side of this equation is less (more) than one, the averaged state of mechanical equilibrium is stable (unstable). Linear relationship was predicted in the experimental work of Putin G.F. et al [8] where the relationship between the Nusselt number and Ra, Gs was determined. Similar to (1) relation was obtained by extrapolation of experimental results for the heat flux through the layer for the value $N u=1$. It is obviously from (1) that for sufficiently large values of Gershuni number $\left(G s>G s_{c r}\right)$ convective instability occurs when heating is from above, i.e. when $R a<0$.

This work presents an attempt to understand the nature of supercritical regimes of thermal vibrational convection in a layer in low-mode approximation that has been never conducted before.

\section{Problem formulation}

We consider an infinite horizontal layer of a viscous incompressible fluid of thickness $h$ between solid horizontal planes (Fig. 1). The coefficients of kinematic viscosity $\nu$, thermal diffusivity $\chi$, the average density $\rho$, the volume coefficient of thermal expansion $\beta$ and the acceleration of gravity $g$ are assumed to be constant. Ideal heat conductivity and constant transverse temperature gradient $\mathbf{A}$ are assumed. Walls have different constant temperatures and the temperature difference is $A h$. The layer undergoes high frequency harmonic vibration along the unit vector $\mathbf{k}$.

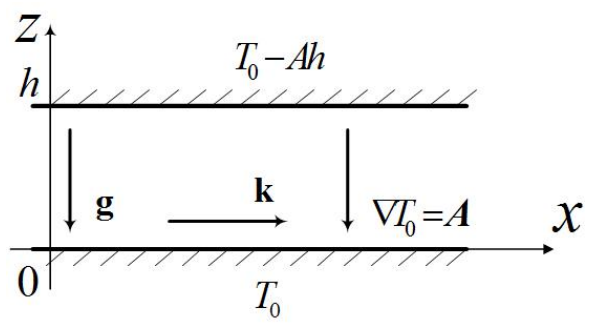

Fig. 1. Geometry of the problem

It is assumed that the vibration frequency is quite high. Therefore, equations of thermal vibrational convection [5] are used to consider the behavior of the fluid. We assume that the liquid moves in the plane. The following characteristic values are introduced: time is $h^{2} \nu^{-1}$, length is $h$ temperature is $A h$, velocity is $\nu h^{-1}$, streamfunction is $\nu$ and pressure is $\rho \nu^{2} h^{-2}$. Dimensionless equations of the thermal vibrational convection, describing the flat convection motion in terms of streamfunction of average motion $\psi$, amplitude of streamfunction of oscillating motion $F$ and average temperature $T$ have the form

$$
\begin{gathered}
\frac{1}{\operatorname{Pr}}\left(\frac{\partial \Delta \Psi}{\partial t}+\frac{\partial \Psi}{\partial x} \frac{\partial \Delta \Psi}{\partial z}-\frac{\partial \Psi}{\partial z} \frac{\partial \Delta \Psi}{\partial x}\right)= \\
\frac{\partial T}{\partial t}+\frac{\partial \Psi}{\partial x} \frac{\partial T}{\partial z}-\frac{\partial \Psi}{\partial z} \frac{\partial T}{\partial x}=\Delta T \\
\Delta F=-\frac{\partial T}{\partial z} \\
-159-
\end{gathered}
$$


It is assumed that the boundaries of the layer are solid and isothermal [4]:

$$
\begin{aligned}
& z=0: \Psi=\frac{\partial \Psi}{\partial z}=F=0, \quad T=1, \\
& z=1: \Psi=\frac{\partial \Psi}{\partial z}=F=0, \quad T=0 .
\end{aligned}
$$

The solution is dictated by three dimensionless numbers: the gravitational Rayleigh number $R a$, the vibration Rayleigh number Gs (the Gershuni number) and the Prandtl number Pr:

$$
\begin{gathered}
R a=\frac{g \beta A h^{4}}{\nu \chi}, \\
G s=\frac{\left(b \beta \Omega A h^{2}\right)^{2}}{2 \nu \chi}, \\
\operatorname{Pr}=\frac{\nu}{\chi} .
\end{gathered}
$$

Equations (2)-(5) have trivial solution that corresponds to the mechanical quasiequilibrium:

$$
\begin{gathered}
\Psi_{0}=0, \\
T_{0}=1-z, \\
F_{0}=\frac{1}{2} z(z-1) .
\end{gathered}
$$

Let us introduce new variables for deviations from this solution. The system of equations (2)-(4) can be rewritten in terms of new variables $\xi, \theta$ and $f$ :

$$
\begin{gathered}
\frac{1}{\operatorname{Pr}}\left(\frac{\partial \Delta \xi}{\partial t}+\frac{\partial \xi}{\partial x} \frac{\partial \Delta \xi}{\partial z}-\frac{\partial \xi}{\partial z} \frac{\partial \Delta \xi}{\partial x}\right)=\Delta \Delta \xi+R a \frac{\partial \theta}{\partial x}+G s\left[\frac{\partial \theta}{\partial x}\left(1+\frac{\partial^{2} f}{\partial z^{2}}\right)+\left(1-\frac{\partial \theta}{\partial z}\right) \frac{\partial^{2} f}{\partial x \partial z}\right] \\
\frac{\partial \theta}{\partial t}+\frac{\partial \xi}{\partial x} \frac{\partial \theta}{\partial z}-\frac{\partial \xi}{\partial z} \frac{\partial \theta}{\partial x}-\frac{\partial \xi}{\partial x}=\Delta \theta \\
\Delta f=-\frac{\partial \theta}{\partial z} .
\end{gathered}
$$

Boundary conditions (5) take the form

$$
z=0,1: \quad \xi=\frac{\partial \xi}{\partial z}=\theta=f=0
$$

\section{Low-mode model}

Let us apply Galerkin method with a minimal set of basic functions to the analysis of nonlinear modes. The following basic functions are used: $\sin (k x) p_{1}(z)$ for $\xi$ and $\cos (k x) p_{1}(z), p_{2}(z)$ for $\theta$. Here $p_{1}(z)$ and $p_{2}(z)$ are mutually orthogonal polynomials of the form

$$
\begin{gathered}
p_{1}(z)=\frac{1}{11} z^{2}(z-1)^{2}, \\
p_{2}(z)=p_{1}^{\prime}(z)=\frac{2}{11}\left(z(z-1)^{2}+z^{2}(z-1)\right), \\
p_{2}^{\prime}(z)=p_{1}^{\prime \prime}(z)=\frac{2}{11}\left((z-1)^{2}+4 z(z-1)+z^{2}\right), \\
p_{1}^{I V}=2 . \\
-160-
\end{gathered}
$$


Polynomial $p_{1}(z)$ was first proposed to approximate the vertical velocity in the layer with solid boundaries [1]. Let us assume that

$$
\begin{gathered}
\xi(x, z, t)=\xi(t) \sin k x \cdot p_{1}(z), \\
\theta(x, z, t)=\theta_{1}(t) \cos k x p_{1}(z)+\theta_{2}(t) p_{2}(z), \\
f(x, z, t)=\theta_{2}(t) f_{2}(z) .
\end{gathered}
$$

Where functions $\xi, \theta_{1}$ and $\theta_{2}$ depend only on time.

Substituting (22) into (14), we obtain the equation for $f_{2}(z)$ :

$$
f_{2}{ }^{\prime \prime}=-p_{2}^{\prime}(z)=-p_{1}^{\prime \prime} .
$$

The following conditions must be satisfied at boundary points:

$$
f_{2}(0)=f_{2}(1)=0
$$

Substituting (20)-(22) into equations (12)-(14), we obtain equations for $\xi, \theta_{1}$ and $\theta_{2}$

$$
\begin{gathered}
\frac{k^{2}+12}{\left(k^{2}+12\right)^{2}+360} \frac{\dot{\xi}}{\operatorname{Pr}}=-\xi+\frac{k\left(R a+G s-\frac{30}{121} G s \theta_{2}\right) \theta_{1}}{\left(k^{2}+12\right)^{2}+360}, \\
\dot{\theta}_{1}=-\left(k^{2}+12\right) \theta_{1}+k \xi+\frac{30}{121} k \xi \theta_{2}, \\
\dot{\theta}_{2}=-\frac{28}{3} \theta_{2}-\frac{2}{3267} k \xi \theta_{1} .
\end{gathered}
$$

Let us introduce new variables:

$$
\theta_{1}=\frac{121 \sqrt{3}}{\sqrt{10}} \vartheta_{1}, \quad \theta_{2}=-121 / 30 \vartheta_{2}, \quad \xi=\frac{\left(12+k^{2}\right)}{k} \frac{121 \sqrt{3}}{\sqrt{10}} \psi, \quad t=\tau /\left(12+k^{2}\right) .
$$

Then system (25)-(27) can be written as

$$
\begin{gathered}
\frac{c}{\operatorname{Pr}} \dot{\psi}=-\psi+\left(r_{g}+r_{v}\right) \vartheta_{1}+r_{v} \vartheta_{1} \vartheta_{2}, \\
\dot{\vartheta}_{1}=-\vartheta_{1}+\psi-\psi \vartheta_{2}, \\
\dot{\vartheta}_{2}=-b \vartheta_{2}+\psi \vartheta_{1} .
\end{gathered}
$$

The following designations are used:

$$
\begin{gathered}
b=\frac{28}{3\left(12+k^{2}\right)}, \quad c=\frac{\left(k^{2}+12\right)^{2}}{\left(k^{2}+12\right)^{2}+360}, \\
r_{g}=R a / R^{*}, \quad r_{v}=G s / R_{l-m}, \\
R_{l-m}=\frac{1}{k^{2}}\left(k^{2}+12\right)\left(\left(k^{2}+12\right)^{2}+360\right) .
\end{gathered}
$$

System (29)-(31) with $c=1$ и $r_{v}=0$ is well-known Lorentz model [9]. Therefore, this system can be regarded as a generalization of the Lorenz model to the case of longitudinal vibration and more realistic conditions on solid walls (sticking in the Lorentz model is absent). There is generalization of the Lorenz model to the case of transverse vibrations [10]. 


\section{Stability of trivial solution}

Equations (29)-(31) have trivial solution that corresponds to the mechanical quasi-equilibrium state:

$$
\psi_{1}=0, \quad \vartheta_{1}=0, \quad \zeta_{1}=0 .
$$

In order to investigate the stability of (38) small perturbations are introduced:

$$
\psi=\tilde{\psi} \exp (\lambda t), \quad \vartheta=\tilde{\vartheta} \exp (\lambda t), \quad \zeta=\tilde{\zeta} \exp (\lambda t) .
$$

Substituting (36) into (29)-(31) and taking into account that we have small-amplitude perturbations, system of homogeneous equations with respect to amplitudes of perturbations is obtained. The homogeneous system of equations has nontrivial solution if its determinant is equal to zero:

$$
\Delta=(\lambda-b)\left(\frac{c}{\operatorname{Pr}} \lambda^{2}-\left(\frac{c}{\operatorname{Pr}}+1\right) \lambda+1-r_{g}-r_{v}\right)=0 .
$$

This equation has the following roots:

$$
\lambda_{1}=-b, \quad \lambda_{2,3}=-\frac{1+\mathrm{P} r^{\prime}}{2} \pm \frac{1}{2} \sqrt{\left(1+\mathrm{P}^{\prime}\right)^{2}+4 \mathrm{P} r^{\prime}\left(r_{g}+r_{v}-1\right)} .
$$

It is clear that root $\lambda_{1}$ is always real and negative. Roots $\lambda_{2}$ and $\lambda_{3}$ can be either complex conjugate with negative real parts or real.

If $r_{g}+r_{v}<1$ then real parts of $\lambda_{2}$ and $\lambda_{3}$ are negative and quasi-equilibrium state is stable for such set of parameters $\left(r_{g}, r_{v}\right)$. If $r_{g}$ is negative and sufficiently large in absolute value (it corresponds to strong heating from above) then $\lambda_{2}$ and $\lambda_{3}$ are complex conjugate. This points to the oscillatory nature of perturbations. Dumping of perturbations takes place under the condition:

$$
r_{g}<-\frac{1}{4} \frac{(\operatorname{Pr}-c)^{2}}{\operatorname{Pr} c}-r_{v}
$$

It is evident that the greater the intensity of the vibration the more intense heating from above is necessary for the occurrence of oscillations.

If $r_{g}+r_{v}>1$ then $\lambda_{2}$ is positive and quasi-equilibrium state becomes unstable. In the case $\left\{\lambda_{2}=0\right\}$ we obtain

$$
r_{g}^{0}+r_{v}^{0}=1 .
$$

Taking into account (32), one can obtain that quasi-equilibrium state becomes neutral stable for

$$
R a+G s=R a_{l-m} .
$$

Thus, the dependence (34) for $G s=0(R a=0)$ defines in low-mode approximation the critical value of the Rayleigh number $R a^{*}=R a_{l-m}-G s$ (the Gershuni number $G s^{*}=R a_{l-m}-R a$ ) above which the perturbation wave number $k$ increases. It is clear from (34) that neutral curve $R a_{l-m}(k)$ has a minimum at $k_{m}=3.214$.

This means that the most dangerous perturbation occurs when the cell size is approximately equal to the thickness of the layer. This value corresponds to the wave number of the critical number:

$$
R a_{l-m}^{*}=1856 .
$$

This value is a good approximation to more accurate values of the critical Rayleigh number $R a_{c} r$ and the critical Gershuni number $G s_{c} r$. 
Comment. System (29)-(31) with $c=1$ и $r_{v}=0$ becomes Lorentz model [9]. Therefore, this system can be regarded as a generalization of the Lorenz model to the case of longitudinal vibration and more realistic non slip boundary conditions on solid walls. The analysis of linear model shows that the occurrence of both gravitational and vibrational convection is predicted with reasonable accuracy. In this model the critical gravitational Rayleigh number and vibrational Rayleigh numbers coincide and they are equal to 1856. This value differs from the exact values of the critical Rayleigh number 1708 [1] and the critical value of the vibrational Rayleigh number (the Gershuni number) 2129 [5] by about 10 per cent. In this model non-slip conditions on the walls are assumed in contrast to the Lorentz model where viscous stresses at the borders are missed.

\section{Stationary finite-amplitude solutions}

The stationary solution of system of nonlinear ordinary differential equations (29)-(31) can be obtained in analytical form. From (30) and (31) one can express $\theta$ and $\zeta$ in terms of $\psi$ :

$$
\vartheta=\frac{b \psi}{b+\psi^{2}}, \quad \zeta=\frac{\psi^{2}}{b+\psi^{2}} .
$$

These expressions were obtained in [11]. By substituting (43) into (29), we obtain the equation of the fifth degree with respect to $\psi$ :

$$
\psi\left(\psi^{4}+\left(2\left(1-r_{v}\right)-r_{g}\right) b \psi^{2}+\left(1-r_{v}-r_{g}\right) b^{2}\right)=0 .
$$

This equation, apart from the trivial solution $\psi_{1}=0$, has four roots:

$$
\begin{aligned}
\psi_{2,3} & = \pm \sqrt{\frac{b}{2}\left[\sqrt{r_{g}^{2}+4 r_{v}\left(r_{g}+r_{v}-1\right)}+r_{g}+2\left(r_{v}-1\right)\right]}, \\
\psi_{4,5} & = \pm \sqrt{\frac{b}{2}\left[-\sqrt{r_{g}^{2}+4 r_{v}\left(r_{g}+r_{v}-1\right)}+r_{g}+2\left(r_{v}-1\right)\right]} .
\end{aligned}
$$

The Gershuni number can not be negative, and the Rayleigh number can be either positive or negative. To analyse equations (44)-(46) it is sufficient to consider the half-plane of parameters $\left(r_{g}, r_{v}\right)$ with $r_{v} \geqslant 0$. Results of the analysis on the basis of (45), (46)) are shown in Fig. 2 solutions by two bifurcation curves (solid and dashed lines).

I . Range of values, where the only real solution is $\psi_{1}=0$ :

$$
\left[\left(0 \leqslant r_{g}<1\right) \cap\left(r_{v}<1-r_{g}\right)\right] \cup\left[\left(r_{g}<0\right) \cap\left(r_{v}<\frac{1}{2}\left(1-r_{g}+\sqrt{1-2 r_{g}}\right)\right)\right] .
$$

II . Range of values where there are three distinct real solutions - trivial, positive and negative $\psi_{3}=-\psi_{2}$ :

$$
\left(r_{v} \geqslant 0\right) \cap\left(r_{g}>1-r_{v}\right) .
$$

III . Range of values where there are five different real solutions - trivial $\psi_{1}=0$, two positive $\psi_{2}, \psi_{4}$ and two negative $\psi_{3}=-\psi_{2}$ and $\psi_{5}=-\psi_{4}$ :

$$
\left[\left(r_{g}<0\right) \cap\left(r_{v}<1-r_{g}\right) \cap\left(r_{v}>\frac{1-r_{g}+\sqrt{1-2 r_{g}}}{2}\right)\right] .
$$




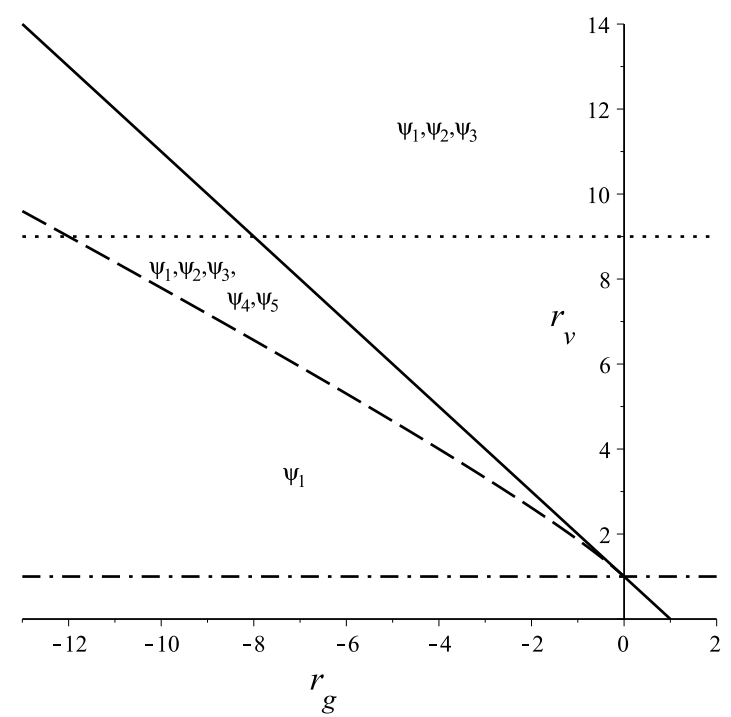

Fig. 2. Map of stationary solutions on the parameter plane $\left(r_{g}, r_{v}\right)$. The trivial solution $\psi_{1}$ exists throughout the parameter plane. Two pairs of solutions $\left(\psi_{2}, \psi_{4}\right)$ and $\left(\psi_{3}, \psi_{5}\right)$ appear or disappear in crossing bifurcation curve (51) (dashed line). It depends on the direction of crossing. Pair of solutions $\left(\psi_{2}, \psi_{3}\right)\left(\left(\psi_{4}, \psi_{5}\right)\right)$ appear or disappear for positive (negative) values of $r_{g}$ in crossing the bifurcation curve $r_{g}+r_{v}=1$ (solid line). This is the result of the fork bifurcation

Areas I and II are connected by the line defined by relations

$$
0 \leqslant r_{g} \leqslant 1, \quad r_{v}=1-r_{g} .
$$

On this line $\psi_{1}=\psi_{2}=\psi_{3}=0$, it means that the solutions $\psi_{2}$ and $\psi_{3}$ are branched out from the trivial solution $\psi_{1}$ during transition from region I to region II.

Areas I and III are connected by the bifurcation curve defined by relations

$$
r_{v}=\frac{1}{2}\left(1-r_{g}+\sqrt{1-2 r_{g}}\right), \quad-\infty<r_{g} \leqslant 0 .
$$

On this line the following conditions are satisfied for nontrivial roots:

$$
\psi_{2}=-\psi_{3}=\psi_{4}=-\psi_{5}=\sqrt{\sqrt{1-2 r_{g}}-1}
$$

Areas II and III are connected by the bifurcation curve defined by relations

$$
\infty<r_{g} \leqslant 0, \quad r_{v}=1-r_{g} .
$$

On this line all five roots are real and the following conditions are satisfied

$$
\psi_{1}=\psi_{4}=\psi_{5}=0, \quad \psi_{2,3}= \pm \sqrt{-2 r_{g}} .
$$

Thus, when heating is from above $\left(r_{g}<0\right)$ then for each $r_{v}>1$ there is a range of Rayleigh numbers $r_{g}$ defined by system of inequalities (47) wherein system (30)-(31) has 5 different stationary solutions. We have $\psi_{4}=\psi_{5}=0$ on the bifurcation curve $r_{g}=1-r_{v}$ (solid line in Fig. 2), $\psi_{4,5}$ are imaginary above this curve and there are 3 real solutions. The system has only the trivial solution $\psi_{1}=0$ below the bifurcation curve defined by equation (51) (dashed line). 
Consider a smooth change of parameters $r_{g}$ and $r_{v}$. It can be represented as a line in the parameter plane $\left(r_{g}, r_{v}\right)$. In crossing these bifurcation curves one or two pairs of solutions appear or disappear. It depends on the direction of crossing.

Typical bifurcation diagrams describing a bifurcation that occurs in crossing bifurcation curves shown in Fig. 2 are presented in Fig. 3 and Fig. 4. Parameters are changed along dotted and dashed-dotted lines. Fig. 3 shows the bifurcation diagram $\psi\left(r_{g}\right)$ for the vibrational Rayleigh number $r_{v}=1$ (it corresponds to the dash-dotted line in Fig. 3). The occurrence of convection is observed when the Rayleigh number increases. This is the result of the soft fork bifurcation when the Rayleigh number is above zero.

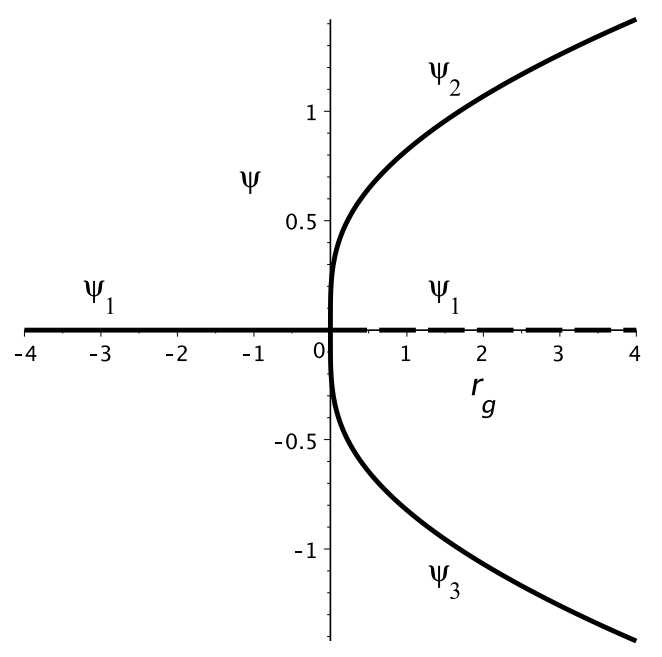

Fig. 3. The diagram of the fork bifurcation that occurs for a smooth variation of the Rayleigh number $r_{g}$ and $r_{v}=1$

Fig. 4 shows the bifurcation diagram $\psi\left(r_{g}\right)$ for the vibrational Rayleigh number $r_{v}=9$ (it corresponds to the dotted line in Fig. 2). One can see that with increasing the Rayleigh number convection occurs in a drastic way at the moment of crossing the bifurcation curve (53). Presented bifurcation diagrams are derived from solutions (45), (46). Constant $b=0.4179$ is obtained from (32) for $k=k_{m}=3.214$.

To determine the stability of solutions presented in Fig. 3 and Fig. 4, numerical solution of the original system of equations (29)-(31) is obtained with the use of the Runge-Kutta-Felberg method (RKF45). The required for the numerical integration parameters $b$ and $c$ are determined from (34) for $k=k_{m}=3.214$ and they are $b=0.4179$ and $c=0.5808$. The Prandtl number is set to $\operatorname{Pr}=c=0.5808$. The initial value of the amplitude of the stream function $\psi$ is close to zero, initial values of $\theta$ and $\zeta$ are calculated from (43). Calculations were carried out for $\left(-20<r_{g}<20\right) \cap\left(r_{v}<20\right)$. They show that solutions $\psi_{2}$ and $\psi_{3}$ are stable but solutions $\psi_{4}$ and $\psi_{5}$ are unstable.

Long arrows in Fig. 3 show the drastic transition from quasi-equilibrium state to one of supercritical states $\psi_{2}$ or $\psi_{3}$. These transitions occur with a continuous stepwise increase in the normalized Rayleigh number $r_{g}$ along the dotted line in Fig. 2 (from left to right). The initial value of the amplitude of stream function $\psi$ is set to the value obtained in the previous step. In the reverse step by step change of the Rayleigh number, sharp damping of the supercritical flow 


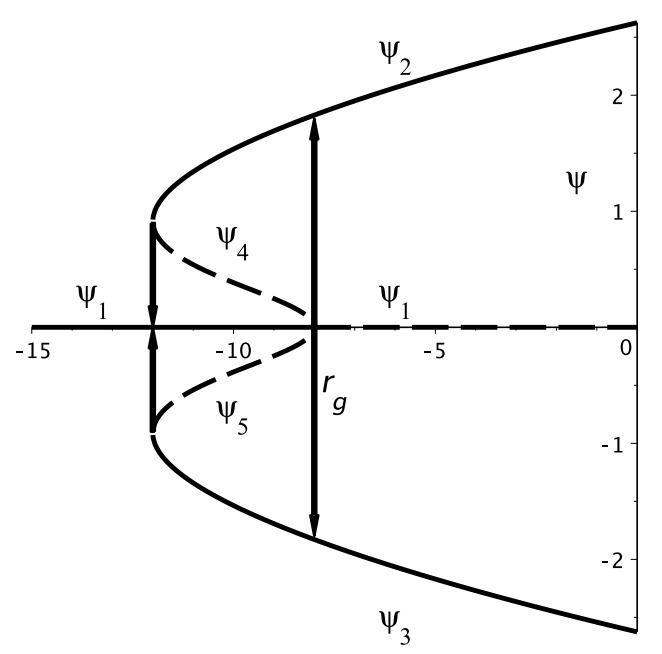

Fig. 4. The bifurcation diagram for $r_{v}=9$

is observed at the moment of crossing the dotted line. When the Gershuni number increases the hysteresis depth also increases.

Calculations were also performed for $\operatorname{Pr}=1$. Bifurcation diagrams are similar to that shown in Figs. 3 and 4.

\section{Stability of stationary finite-amplitude solutions}

Numerical integration for various Prandtl numbers $0<\operatorname{Pr}<10^{2}$ shows that solutions $\psi_{4}$ and $\psi_{5}$ are always unstable. Solutions $\psi_{2}$ and $\psi_{3}$ are unstable for sufficiently large values of Prandtl, Rayleigh and Gershuni numbers. Let us consider small perturbations

$$
\psi^{\prime}=\psi_{2}+\tilde{\psi}_{2} \exp (\lambda t), \quad \vartheta^{\prime}=\vartheta_{2}+\tilde{\vartheta}_{2} \exp (\lambda t), \quad \zeta^{\prime}=\zeta_{2}+\tilde{\zeta}_{2} \exp (\lambda t) .
$$

Since $\psi_{3}=-\psi_{2}$ it is sufficient to consider only the stability of state $\psi_{2}$. Hereinafter the subscript is omitted.

Substituting (55) into (29)-(31), we obtain the system of linear equations for amplitudes of perturbations. Setting the determinant of the system matrix equal to zero, we obtain a cubic equation for $\lambda$ :

$$
\begin{aligned}
\lambda^{3}+\left(b+1+\mathrm{P}^{\prime}\right) \lambda^{2}+\mathrm{P}^{\prime} & {\left[b+1+\frac{b+\psi^{2}}{\mathrm{P} r^{\prime}}-b \frac{\left(b+\psi^{2}\right)\left(r_{g}+r_{v}\right)+r_{v}(b+1) \psi^{2}}{\left(b+\psi^{2}\right)^{2}}\right] \lambda+} \\
+\operatorname{Pr}^{\prime} & {\left[\left(b+\psi^{2}\right)+b \frac{\left(r_{g}+r_{v}\right)\left(\psi^{4}-b^{2}\right)+r_{v} \psi^{2}\left(\psi^{2}-3 b\right)}{\left(b+\psi^{2}\right)^{2}}\right]=0 . }
\end{aligned}
$$

The lack of vibrations $\left(r_{v}=0\right)$. In this case, the stationary solutions (45) take the form:

$$
\psi= \pm \sqrt{b\left(r_{g}-1\right)}
$$

Substitution of this solution into (56) gives

$$
\lambda^{3}+\left(b+1+\mathrm{Pr}^{\prime}\right) \lambda^{2}+b\left(\mathrm{P} r^{\prime}+r_{g}\right) \lambda+2 \mathrm{P} r^{\prime} b\left(r_{g}-1\right)=0 .
$$


This equation has one negative and two complex conjugate roots when $r_{g}>1$. Real parts of complex conjugate roots for $r_{g}>1$ and small or moderate super-criticalities are also negative. Equation (58) is similar to that obtained and analyzed by E. Lorenz [9]. It was shown that by equating the product of coefficients at $\lambda^{2}$ and $\lambda$ to the constant term, one can get the criteria of neutral stability of oscillating disturbances [9]. In our case such equation has the form

$$
\left(b+1+\mathrm{P} r^{\prime}\right)\left(\mathrm{P}^{\prime}+r_{g}\right)=2 \mathrm{P} r^{\prime} b\left(r_{g}-1\right) .
$$

The solution of equation (59) with respect to $r_{g}$ gives a simple expression for the critical value of $r_{g}$ above which the instability of steady convection occurs:

$$
r_{g}^{*}=\frac{\operatorname{Pr}(\operatorname{Pr} / c+b+3)}{\operatorname{Pr}-c(b+1)} .
$$

Thus if $\operatorname{Pr}<c(b+1) \approx 0.8$ then not positive value of $r_{g}$ satisfies $(60)$ and steady convection regime (57) is always stable. If $\operatorname{Pr}>c(b+1)$ then steady convection is unstable with respect to oscillating disturbances for sufficiently large Rayleigh numbers.

Numerical study of stability in the general case $r_{g} \neq 0, r_{v} \geqslant 0$. Now we consider the stability of periodic steady solutions with wave number $k_{m}$. We perform the numerical analysis of equations (56) with fixed values of the Prandtl number $\operatorname{Pr}=1,3,5$ and 10, incrementing small perturbations $\lambda$. Analysis of coefficients at $\lambda, \lambda^{2}$ and constant term in equation (56) shows that they all are non-negative for solutions $\psi_{2,3}$.

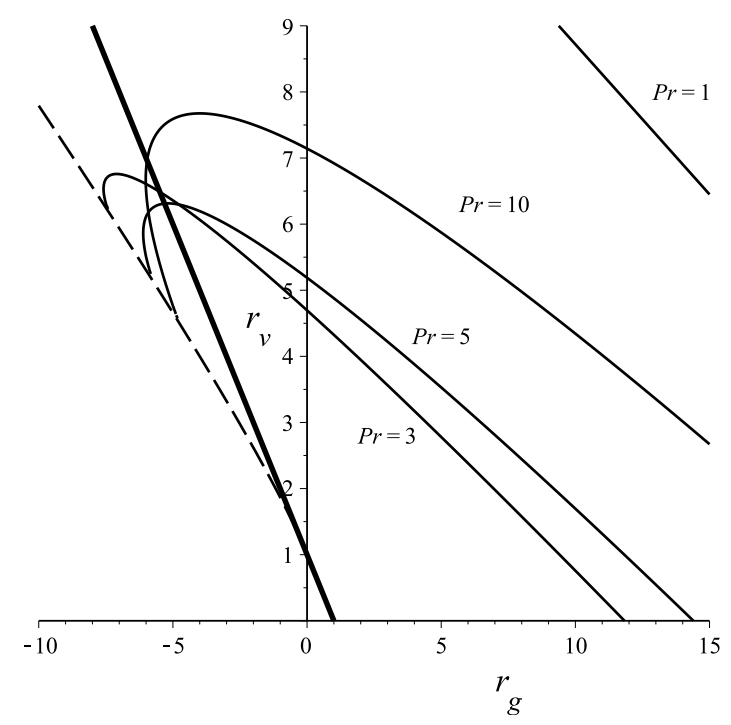

Fig. 5. Areas of stability of supercritical solution $\psi_{2}$ with respect to oscillatory disturbances on the parameter plane $\left(r_{g}, r_{v}\right)$ for various values of the Prandtl number. Bifurcation curves are marked by thin lines. The oscillatory instability appears in crossing these curves. The solid line and the dashed line correspond to the bifurcation curves shown in Fig. 2

This means that, as in the case above $r_{v}=0$, the equating of the product of coefficients at $\lambda$ and $\lambda^{2}$ to constant term leads to the equation that is similar to (59). This gives the stability criteria of solutions $\psi_{1,2}$ with respect to oscillatory disturbances. The equation is rather cumbersome, and it is not presented here. The results of the numerical analysis of the equation 
are presented in Fig. 5 in the form of bifurcation curves (thin solid lines) for various values of the Prandtl number. First we consider the case $\operatorname{Pr}=5$. In accordance with (60), oscillatory instability occurs when the normalized Rayleigh number $r_{g}=14.4$ in the case $r_{v}=0$. When $r_{g}=0$, oscillatory instability occurs if the normalized Gershuni number $r_{v}$ exceeds the critical value $r_{v}^{\prime}=5.2$. The figure shows that in the case of heating from above, i.e., when $r_{g}<0$, the stability region of supercritical steady solutions $\psi_{2,3}$ is defined by the Gershuni number and the Rayleigh number. When $1<r_{v}<5$ the smooth cyclic variation of the Rayleigh number leads to drastic hysteretic transitions similar to that shown in Fig. 4.

As the normalized Rayleigh number $r_{g}$ undergoes smooth cyclical changes, the corresponding path is a horizontal line on the parameter plane. The normalized Rayleigh number should consistently cross solid and dashed lines but should not cross a thin line. Fig. 5 shows that for all considered values of the Prandtl number such situations are observed for $1<r_{v}<4.5$ when the heating is at the top $\left(r_{g}<0\right)$. This is supported by the direct numerical integration of the original system of equations (29)-(31). The obtained bifurcation diagrams are similar to that shown in Fig. 4.

\section{Conclusion}

The low-mode model of the thermovibrational convection in an infinite plane layer of incompressible viscous fluid confined between horizontal solid isothermal planes has been considered. The layer undergoes longitudinal vibrations. In the limiting case of the lack of vibrations the model is similar to the well-known Lorenz model. The results of linear stability analysis of the model are in good agreement with the results of the linear stability analysis of complete equations of thermal vibrational convection.

It is shown that the model predicts the drastic excitation of stationary supercritical vibrational convection in the case of heating from the top. Cyclic variation of the gravitational Rayleigh number leads to hysteretic transitions between stationary solutions.

The study of linear stability of the supercritical flows shows that for all considered Prandtl numbers the region of stability decreases with increasing the Prandtl number. However, there are regions where drastic hysteretic excitation of supercritical convection is possible.

The work was supported by the Perm National Research Polytechnic University under an internal university grant.

\section{References}

[1] G.Z.Gershuni, E.M.Zhuhovitskii, Convective stability of incompressible fluids, Israel Program for Scientific Translations, Jerusalem, 1976.

[2] A.V.Getling, Reylaigh-Benare Convection: Structures and Dynamics, World Scientific, 1998.

[3] V.K.Andreev, V.B.Bekezhanova, Stability of non-isothermal fluids, J. Appl. Mech. Tech. Phys., 54(2013), no. 2, 171.

[4] A.N.Sharifulin, Supercritical vibro-thermal heat transfer in plane layer of flued in nongravity. Methods of aerodynamics and heat mass transfer in technological prosseses, Tomskij Gos. Univ., Tomsk, 1984 (in Russian). 
[5] G.Z.Gershuni, D.V.Luybimov, Thermal vibrational convection, Wiley \& Sons, 1998.

[6] I.I.Ryzhkov, On the Boussinesq Approximation in the Problems of Convection Induced by High-Frequency Vibration, Journal of Siberian Federal Univerity. Mathematics \& Physics, 3(2010), no. 4, 433.

[7] V.Shevtsova, I.I.Ryzhkov, D.E.Melnikov, Y.A.Gaponenko, A.Mialdun, Experimental and theoretical study of vibration-induced thermal convection in low gravity, J. Fluid Mech., 648(2010), 53 .

[8] M.P.Zavaryikin, I.I.Zorin, G. F.Putin, Conserning thermal convectional instability in vibrational field, Dokl. AN SSSR, 303(1988), 309.

[9] E.N.Lorenz, Deterministic nonperiodic flow, Journal of the atmospheric sciences, 20(1963), 130.

[10] D.V.Lyubimov, M.A.Zaks, Two mecanism of the transition to chaos in finite-dimension models of convection, Physics D, 9(1983), 52 .

[11] A.I.Nikitin, A.N.Sharifulin, Concerning the bifurcations of steady-state thermal convection regimes in a closed cavity due to the Whitney folding-type singularity, Heat Transfer. Soviet Research, 21(1989), no. 2, 213.

\section{Маломодовая модель тепловой вибрационной конвекции в горизонтальном слое при наличии продольных вибраций}

Вадим А. Шарифулин

Пермский национальный исследовательский политехнический университет Поздеева, 11/В, Пермь, 614990

Россия

В маломодовом приближении исследованъ устойчивость и бибуркации надкритической термовибрационной конвекиии в горизонтальном слое жидкости между нагретыми до различной температуры изотермическими твердыми границами при наличии продольных вибраций. В области устойчивости надкритической конвекции аналитически получены бифуркационные диаграммы надкритических режимов, анализ которых показал, что при подогреве сверху вибрации могут приводить к жесткому типу возникновения конвекции, сопровождающемуся гистерезисом между стационарными состояниями. Область гистерезиса по числу Рэлея увеличивается с увеличением числа Гершуни. В рамках предложенной модели проведено численное исследование линейной устойчивости надкритических вибрационн-конвективных течений для интервала чисел Прандтля $1 \leqslant \operatorname{Pr} \leqslant 10$. Расчеть показали что хотя область их устойчивости при увеличении числа Прандтля и уменъшается, при всех рассмотренных его значениях из указанного интервала имеются области, где возможно жесткое гистерезисное возбуждение стационарной вибрачионной конвекции.

Ключевые слова: дифференииальные уравнения, задача Коши, расщепление, устойчивость, сходимость. 